\title{
A Framework for Defining Scientific Concepts in Science Education
}

\author{
Chee Leong Wong \\ National Institute of Education, Nanyang Technological University, \\ 1 Nanyang Walk, Singapore 637616 \\ alphonsuswong@gmail.com \\ Hye-Eun Chu \\ Corresponding author, School of Education, Faculty of Arts, Macquarie \\ University, NSw, 2109, Australia \\ hye-eun.chu@mq.edu.au
}

\author{
Kueh Chin Yap \\ National Institute of Education, Nanyang Technological University, \\ 1 Nanyang Walk, Singapore 637616 \\ kcyap47@gmail.com
}

Received: 4 September 2020 | Revised: 12 November 2020 |

Accepted: 18 November 2020

\begin{abstract}
Studies have shown that inadequate definitions of scientific concepts could complicate the learning of science and could prevent students from understanding the definitions of scientific concepts. The article provides a framework for defining scientific concepts in primary, secondary, and university education by proposing teachers draw attention to five common features of a definition: object/system, nature/characteristics, cause/effect, mathematical expression/equation, and condition/ reference frame that can help students to consider four dimensions, including, 'comprehensiveness,' 'precision,' 'consistency,' and 'circularity' when learning science concepts. This framework can be used by science teachers to guide students to analyze and redefine scientific concepts in the classroom and may be especially beneficial for students in Asian countries where teachers and students often rely on rote memorization as a strategy
\end{abstract}


for learning scientific concepts. We conclude by describing the need for future studies in educational contexts in Asian countries where pedagogical strategies have traditionally emphasized memorization of science concepts.

\section{Keywords}

circularity - definition - framework - heat - weight

\section{Introduction}

It may seem impossible to establish a comprehensive framework that can guide scientists and science educators to define scientific concepts. Scientists focus on developing definitions that are accurate, whereas science educators are also concerned with whether students can understand these definitions. The intended purpose of a definition (how it will be used) can also subtly differ among scientists within the same discipline as well as among scientists from different disciplines. Specifically, Margenau (1942) suggested that there should be several different methods to define various scientific concepts, such as speed, electron, and mass. He explained that there were many different ways of defining a concept (e.g., mass) for different purposes. As a result, he proposed that in a general sense there should be two different types of definitions: constitutive and operational, reflecting both theoretical knowledge and experimental procedures.

We can anticipate difficulties or disagreements in implementing a framework for defining scientific concepts. Over the years, and in recent times still, there have been disagreements over many definitions in the fields of biology, chemistry, and physics. In biology, there has been a long philosophical debate on the definition of life (Trifonov, 2012). In chemistry, there has been a continuous debate on the definition of the hydrogen bond due_to the emergence of new experimental and theoretical evidence (Arunan et al., 2011). In physics, current disagreement over the definition of entropy can be illustrated by the existence of at least eight perspectives (Swendsen, 2011). Disagreements over definitions can be beneficial to the development of science by providing more perspectives; unfortunately, the multiple definitions that result can lead to miscommunication among scientists. Nevertheless, a minimal and simple framework for the definition of scientific concepts seems necessary in science education. 
According to Bunge (1998), definitions must be formally correct and materially adequate in both the epistemological and the pragmatic sense. $\mathrm{He}$ proposed that these definitions should satisfy the formal conditions of consistency, exchangeability, and non-creativity. However, these conditions were initially identified by Suppes (1957) for ideal mathematical concepts; it is not straightforward to apply these conditions to real-world scientific concepts that may have numerous anomalies or exceptions. In addition, Bunge's schemes are neither comprehensive nor practical, because he mainly proposed a general need for theoretical and empirical information, without any specific features of definitions. One way to identify such features is to look at the definitional problems that have plagued scientists and science educators: precision, consistency, circularity, and comprehensiveness (Galili \& Lehavi, 2006; Quílez, 20o9; Strömdahl, 2012).

The problem of precision in defining scientific concepts, which is sometimes described as 'ambiguity' or 'vagueness,' can also impede understanding (Keller, 2005; Quílez, 2009; Williams, 1999). Keller (2005) observes that there are problems of ambiguity in the definition of the term gene, and that they can be compounded by the multiplicity of different meanings of gene in various contexts. As another example, the concept of chemical affinity did not have a precise definition; it was historically thought to be a kind of gravitational force (Quílez, 2009). Currently, the International Union of Pure and Applied Chemistry (IUPAC, 2014) defines affinity of reaction as the 'negative partial derivative of Gibbs energy with respect to extent of reaction at constant pressure and temperature' (p. 46). In other words, this chemical concept is now defined in terms of a verbal form of a mathematical equation.

Scientific concepts are often inconsistently defined in different fields or within a field (Doige \& Day, 2012; Strömdahl, 2012). This problem is sometimes discussed in terms of the linguistic concept of polysemy, which means that one word is used with two or more related meanings (Strömdahl, 2012). For instance, studies have shown that students learning biology concurrently with physics and/or chemistry are commonly confused by the concept of energy (Gayford, 1986). Based on Lancor's (2014) analysis, biology textbooks commonly adopt language indicating that energy is lost from systems (usually open systems). This is in contrast to physics textbooks, which emphasize the conservation of energy in closed systems. Thus, students can have difficulty coping with the multiple definitions of this scientific term.

The problem of circularity in defining scientific concepts can result in definitions that do not provide useful knowledge (Galili \& Lehavi, 20o6; Harwood, 2009; Laszlo, 1999). In chemistry, for example, the problem of circularity poses 
a difficulty for students as the concept of acid is defined in terms of the base concept and vice versa (Laszlo, 1999). Other examples of circularity (or dual concepts) in chemistry are electrophile-nucleophile, substrate-reagent, and receptor-agonist (Kaya \& Erduran, 2013).

Finally, a definition of a scientific concept should also be comprehensive, representing all its essential features (Huang, 2009; Portin, 2009). However, it has not been clear to scientists and science educators what these essential features should be so that definitions can be comprehensive, or how they can be determined. In Philosophical Investigations, Wittgenstein (1953) argued that it is inherently difficult or perhaps impossible to define some terms. Discussing consistency in the intended meanings of a word, he introduced the concept of family resemblance, which denotes that the instances of a term being defined (e.g., game) constitute a family whose members do not have any single common feature, but instead have subsets of features that are shared by some (but not all) members of the family. Similarly, we do not expect all scientific concepts must have the same common features among definitions, however, we propose that it is possible to identify relatively common features among definitions of scientific concepts.

Importantly, educational studies have found that students may have difficulty with definitions or vocabulary loads. In Groves's (1995) analysis of vocabulary loads of selected secondary science textbooks, Modern Chemistry had 2,950 scientific terms, Biological Science had 1,899 terms, and Physics had 1,538 terms. The number of features in a definition can further increase the memory loads of students. Therefore, there should be a reduction in the emphasis on memorizing definitions of scientific terms, because students do not find it meaningful to remember so many terms (Groves, 1995). In this paper, we do not delve into the potential benefits of memorizing definitions of scientific concepts and nor do we intend to debate whether students in Asian countries tend to memorize definitions of scientific concepts. However, it is interesting to note that while PISA results show that students in Asian countries are relatively better in science contents, they score less well on items assessing science process (Khine, 2015). We hope that the proposed framework can provide students in countries where memorization has been encouraged a new method for approaching the study of scientific concepts that can improve not only understanding of science contents, but also science process and critical thinking skills. We believe that students can greatly benefit from an approach that views knowledge learning, including the learning of theoretical definitions of terms, should be constructed or co-constructed by the students with the facilitation of the teachers. In other words, the learning of a definition is 
more meaningful if students are guided to construct the definition or concept (Wong, Chu, \& Yap, 2014), rather than memorizing.

\section{Common Features of Definitions}

Each scientific concept is unique and there are no fixed categories in which scientists can characterize scientific concepts. However, in characterizing the concept game, Wittgenstein (1953) wrote that '[w]e do not know the boundaries because none have been drawn. To repeat, we can draw a boundary for a special purpose' (p. 33). Similarly, to develop a framework for defining scientific concepts, we propose to draw boundaries for five common features of definitions: broader and flexible categories that are neither fixed nor clear-cut, but fuzzy-edged. This is in contrast to Wittgenstein's fixed categories in defining members of a family: 'build, features, color of eyes, gait, temperament' (p. 32). We will discuss five common features of scientific definitions: object/system and its nature/characteristics, cause/effect, mathematical expression/equation, and condition/reference frame. It is important to identify these features of definitions such that they can be systematically analyzed in order to examine the related definitional problems.

In general, a scientific concept should be defined by specifying aspects of its characteristics, attributes, or properties that are common to most members of that concept 'family' (e.g., genes) but not common to all other scientific concepts (e.g., planets). Properties, for example, can be further classified into biological, chemical or physical properties. This is an important feature of definitions as it specifies the nature of a scientific concept. Moreover, scientists have to define numerous objects or systems and relate them to existing scientific concepts (Benfey, 2006). This is another important feature of science, because in order to conduct a scientific investigation, we may need definitions of living and non-living entities such as animals, plants, chemicals, subatomic particles, and planets. Thus, we do not simply define length or temperature (nature/characteristic), but the 'length of an object' or 'temperature of a system.'

Scientific concepts can be inter-connected in terms of objects' properties and causal relations (Humphreys, 1999). Definitions of scientific concepts are often specified using words such as caused by, due to, arising from and effect of; thus, suggesting another feature of definition, cause/effect. For instance, the nature of weight is commonly defined as a gravitational force, and some define weight as being 'due to' gravity or a gravitational field. Alternatively, the nature 
of weight is defined in some textbooks as a contact force, and thus the cause of weight in this case can be due to a gravitational field and acceleration of an elevator. Moreover, in Coelho's (2010) analysis of physics textbooks, force is commonly defined as 'the cause of acceleration'; both force and acceleration can be operationally measured. In short, this feature of definition is verifiable through observations or experiments.

Currently, a scientific term can be defined in terms of a mathematical expression or equation. The IUPAC (2014) defines entropy as 'the change in which is equal to the heat brought to the system in a reversible process at constant temperature divided by that temperature' (p. 517); a verbal form of the equation, $\Delta S=\Delta Q / T$. On the other hand, the IUPAC's (2014) definition of a hydrogen bond specifies its associated energies as being usually lesser than $20-25 \mathrm{~kJ} \mathrm{~mol}^{-1}$, which means that it can be more precisely defined in terms of a mathematical expression. In a similar sense, scientists can define (visible) light as a transverse electromagnetic wave or in terms of Maxwell's equation; better still, based on the IUPAC's (2014) definition of visible, the wavelength could be specified as 'between $400 \mathrm{~nm}$ and $760 \mathrm{~nm}$ ' (p. 1596).

Finally, Suppe (2000) proposed that definitions are analytic specifications of meaning and that we should specify a condition of validity. That is, a scientific concept may only exist under certain conditions of validity or physical conditions. In general, a condition of a scientific concept could also be a condition of measurement, an environmental condition, or a condition of applicability such as a frame of reference. For example, the IUPAC (2014) defines batch in analytical chemistry as a quantity of material which is assumed to be produced 'under uniform conditions' (p. 151). Moreover, the IUPAC (2014) states that 'diffusion coefficients may be defined depending on the choice of the frame of reference' (p. 413). Thus, we suggest condition/reference frame as another feature of a scientific definition.

The aforementioned five features of definition can be related to the four definitional problems/considerations already discussed above (comprehensiveness, precision, consistency, and circularity). ${ }^{1}$ Thus, we propose to improve

1 The "five features of a definition" and "four considerations" are based on a PhD thesis titled "A framework for defining physical concepts" (Wong, 2014). The framework was developed by analyzing over 1000 journal articles from biology, chemistry, physics, mathematics, and philosophy, 5 oo physics textbook definitions, and marking schemes (rubrics or grading criteria) on definitions. That is, we can also use the framework to analyze textbook definitions, marking schemes on definitions, and how alternative conceptions were resulted from definitions that have issues of comprehensiveness, precision, consistency, or circularity. However, additional analysis was carried out on more articles and definitions from biology, chemistry, physics, mathematics, and philosophy. Specifically, there were research on IUPAC's (2014) 
current definitions of scientific concepts by resolving definitional problems and by analyzing the common features of definitions.

\section{A Framework for Defining Scientific Concepts}

In this framework, we first identify some of the common features in the definitions of a scientific concept, such as object/system, nature/characteristics, cause/effect, mathematical expression/equation, and condition/reference frame. However, we may not be able to completely resolve the four definitional problems: comprehensiveness, precision, consistency, and circularity. This section will discuss each of the latter in terms of each of the former. This is not a prescriptive framework in which we have to always utilize all five common features of definition.

\subsection{Comprehensiveness}

In seeking a comprehensive definition of a scientific concept, science educators must determine the number of features considered reasonable for adoption. For example, it is possible to develop a comprehensive definition of temperature that includes the following common features: an ideal gas (object/system), average kinetic energy (nature/characteristics), tendency to transfer energy (cause/effect), $T=P V / n R$ (mathematical expression/equation), and thermal equilibrium (condition/reference frame). We will discuss each of these five features using selected textbook definitions of temperature:

Object/system: The definition of a scientific concept should usually specify an ideal object, a real object, or an idealized system; this feature should be identified for most (if not all) scientific concepts. Temperature can be defined in terms of the characteristics of an ideal gas, such that it does not depend on the properties of any particular substance. In general, nonlinear properties related to temperature can be dependent on the object or system specified. Although we may specify the temperature at a particular location in a room, it is scientifically meaningless to define the temperature of a 'point-particle' to which there is no physical significance.

Nature/characteristics: The main purpose of a definition is to determine the nature or the meaning of a term. It is essential to specify 'what it is' or describe possible characteristics of a concept using a definition. One common definition of temperature is a measure of the average kinetic energy of the molecules

definitions and definitions of gene, but a section on "defining the concept of gene" was omitted because it may appear very complicated to many readers. 
within a substance. This specification of meaning relates the concept of temperature to the random motion of molecules.

Cause/effect: A definition of a scientific concept can specify a cause or effect. As an example, temperature can be related to kinetic energy that is caused by the vibrations and rotations of molecules. However, some scientists may prefer to include effect in a definition. Baierlein (1990) proposed to relate temperature to a system's tendency to transfer energy. In this sense, a system with a higher temperature has the effect, or (increased) tendency, to transfer thermal energy. In other words, the temperature of a system 'causes' the transfer of thermal energy, if it is in thermal contact with a system of lower temperature.

Equation/mathematical expression: An equation for temperature may provide either a macroscopic or a microscopic perspective to science students. From a macroscopic point of view, the temperature of an ideal gas can be defined by the equation $T=P V / n R$. It can be dependent on macroscopic quantities such as a partial pressure of a gas. From a microscopic view, the temperature of an ideal gas is proportional to the average molecular kinetic energy and can be represented by the equation $\left.3 k T / 2=1 / 2 m<v^{2}\right\rangle$. It is related to the rootmean-square velocity of gas molecules.

Condition/reference frame: For a definition of temperature, the macroscopic condition could be specified as thermal equilibrium. That is, a system has the same temperature throughout all regions within itself. Microscopically speaking, Alonso (1990) selected to specify statistical equilibrium: If a system is relatively large and not in statistical equilibrium, local temperature may be associated with smaller volume, such that it is considered to be approximately in statistical equilibrium. The specification of a condition is important inasmuch as it helps in understanding the validity of the concept.

There may not be agreement on whether all of the aforementioned features are equally important to the definition of a scientific concept. It could actually be overwhelming for students to include all these features for most scientific concepts. However, these features can still be helpful as guides by allowing science educators to systematically determine which features can be considered reasonable and relevant for a definition. Alternatively, we may identify two or three primary features, which will vary depending on the scientific concepts at hand; the remaining features can be classified as secondary.

A definition of a scientific concept may sometimes be considered noncomprehensive even if it includes all five of the aforementioned features, because it is not necessarily the case that specifying an example of each feature can be considered sufficient for a definition. For example, Huang's (2009) definition of metabolic syndrome includes four 'conditions': insulin resistance, visceral adiposity, atherogenic dyslipidemia, and endothelial dysfunction. 
Similarly, the nature of energy as a scientific concept at the high school level can include characteristics like conservation, transfer, transformation, and degradation (Duit, 2014). However, at the university level, physicists may also include the quantization of energy. In general, scientists prefer a comprehensive definition that represents the nature of a concept as accurately as possible, depending on current scientific knowledge. In contrast, science educators' notion of a 'comprehensive definition' depends on their students' background. Thus, comprehensiveness is contextual; what counts as a comprehensive definition in an introductory course, might not be considered comprehensive in an advanced graduate course.

\subsection{Precision}

Pascal (1657) explained that the freedom to assign a meaning to a scientific term can cause confusion when the term has at least two different meanings. Ideally, the terms used in defining a scientific concept should also be precise such that they do not indicate multiple meanings. According to Ruchlis (1964), a precise definition is related to the exactness of a statement and expresses the current state of knowledge. The degree of precision of a statement is also determined by the context and the reader. In chemistry, it has been proposed that a hydrogen bond be defined as an attractive interaction between a hydrogen atom and a molecule, or a molecular fragment $X-H$ in which $X$ is more electronegative than $H$ (Arunan et al., 2011). However, this definition (although meaningful) may be considered less precise when the term 'electrostatic interaction' is replaced by 'attractive interaction' (in that gravitational interaction, for example, is also an attractive interaction).

Williams (1999) proposed that there should be improvements in the precision of language such that it is similar to the precision of mathematics or measurements. Strictly speaking, however, many problems of precision cannot be completely resolved. Feynman et al. (1963) suggested that features of definition such as an object or equation may involve idealizations and approximations that make achieving a perfectly precise definition difficult or improbable. Likewise, Einstein (1921) explained that 'as far as the propositions of mathematics refer to reality, they are not certain; and as far as they are certain, they do not refer to reality' (p. 233). Simply phrased, we should not assume that mathematical equations can represent nature exactly. In general, imprecision in a definition can be traceable to at least one of its features. In what follows, we will illustrate possible problems of precision by discussing the definitions of weight.

Object/system: The precision of a definition depends on the description of an object within that definition. For example, the weight of a human body is a more precise concept than the weight of a body. Similarly, Serway (2003) 
prefers to define weight as a property of a system consisting of a body and the earth. This definition could be criticized because it implies that a body on the moon has no weight; to address this, some may prefer making it less precise by changing 'the earth' to 'a nearby celestial body.' This can, in turn, lead to the question of whether other celestial bodies farther away might have any effect on weight (by affecting the gravitational force). However, one may argue that this definition cannot be experimentally verified or theoretically calculated in a precise manner.

Nature/characteristics: The nature of weight can be defined in terms of a gravitational force. Some scientists, in order to be more precise, prefer the phrase resultant gravitational force instead of gravitational force; they rationalize that weight is not simply derived from the gravitational field of the earth, because that field can be altered by the gravitational fields of the moon and the sun.

Cause/effect: Giordano (2010) defined weight as existing 'due to the gravitational attraction of the earth (or whatever planet, etc., on which the object is located)' (p. 59). For a theoretical definition in university textbooks, precise technical terms such as gravitational field could be used in place of looser ones like gravitational attraction.

Mathematical expression/equation: The precision of an equation is dependent on the scientific theory that it is based on, and possibly, some additional assumptions. For example, if weight is defined near the surface of the earth, it can be represented by the equation, $W=m g$, where $g$ is acceleration due to gravity, assumed to be relatively constant. However, some textbook authors define weight as a gravitational force and represent it by the equation $W=$ $G M m / r^{2}$; this definition is applicable beyond the surface of the earth. This equation can also be considered more accurate because the calculated weight is closer to the measured weight.

Condition/reference frame: French (1971) stated that a weighing procedure can be carried out under 'any conditions.' One may ask whether the weight of an object can be measured when the ground is vibrating. To be precise, Halliday et al. (2001) state that, "[t] he weight of a body must be measured when the body is not accelerating downward vertically relative to the ground' (p. 96). Alternatively, some scientists specify the condition of weighing in which the object and weighing scale are in static equilibrium in an inertial frame.

In general, the concept of weight may be imprecisely defined by means of idealizations or approximations. For instance, the object of the weight is sometimes limited only to the earth or a nearby celestial body; the nature of weight can be specified as a gravitational force instead of a resultant gravitational force; the cause of weight may be specified as a 'gravitational field' without 
including the rotation of the earth; the equation of weight may be represented by $W=G M m / r^{2}$ without the inclusion of the effects due to the presence of sun, moon, or rotation of the earth; and the condition of weighing may be specified as stationary in an inertial frame. However, the concept of an inertial frame is itself idealized because the observer is assumed to be moving at constant velocity and there is no resultant force acting on this observer.

Although we need to reduce problems of precision in defining scientific concepts, we should also be aware that it is impossible to define physical concepts with ultimate precision (Feynman et al., 1963). Scientists should check whether the features of a definition, such as its object, nature, and cause, are precisely specified. In addition, an equation should be specified in such a way that the concept can be operationalized without ambiguity. Nevertheless, the precision of a definition should not be as such to hinder its use in science teaching.

\subsection{Consistency}

Scientific concepts may be defined differently within the same discipline. Morrison (1999), for example, observes that definitions of weight are inconsistent across introductory physics textbooks. Specifically, the (nature of the) weight of an object can be defined as a gravitational force or as a contact force, depending on the textbook. However, students may have difficulty coping with these two different definitions of weight that can be found within the same textbook (e.g., Hewitt, 2006).

Furthermore, scientific concepts may have multiple definitions in different scientific disciplines. For example, the term heat can be defined differently in biology, chemistry and physics. The IUPAC (2014) defines heat as the 'energy transferred from a hotter to a cooler body due to a temperature gradient' (p. 661). Romer (2001), a former editor of the American Journal of Physics, suggested that heat should be defined as 'not a noun.' In other words, heat can be defined as a transfer of energy (process) instead of the energy transferred (noun). Thus, the framework presented here can help pinpoint possible problems of consistency by comparing definitions of heat and other concepts across fields. Below, we will elaborate on problems of consistency from the perspective of the five features using common definitions of heat.

Object/system: In general, authors of biology textbooks define heat as situated within a body (e.g., Campbell \& Reece, 2008). In chemistry, the IUPAC (2014) specifies that heat is transferred 'from a hotter to a cooler body' (p. 661); similarly, in physics, heat may be defined as being transferred 'between a system and its surroundings.' Essentially, then, the definitions of heat have placed it either 'inside an object' or 'between two systems.' 
Nature/characteristics: The nature of heat is defined in different fields as the 'transfer of energy,' 'energy transferred,' or 'molecular kinetic energy' (Doige \& Day, 2012). For example, authors of biology textbooks define heat as the 'total kinetic energy' contained in an object (Campbell \& Reece, 2008), while the IUPAC (2014) defines the nature of heat as 'energy transferred'; in contrast, Giancoli (2005), a physics textbook author, defines heat as 'a transfer of energy - not to energy itself' (p. 385). Similarly, Pushkin (1997), a physicist, prefers to define heat as a 'process of energy transfer'; this is in contrast to definitions of heat being a form of energy, as described in some physics textbooks.

Cause/effect: The IUPAC (2014) specifies the cause of heat as 'temperature gradient' (p. 661). In Zemansky and Dittman's (1981) Heat and Thermodynamics, heat is defined as the energy 'which is transferred between a system and its surroundings by virtue of a temperature difference only' (p. 70). Similarly, in Doige and Day's (2012) study, the cause of heat, commonly known as temperature difference, can be found in chemistry and physics textbooks. However, authors of biology textbooks may not specify 'temperature difference' because this cause is not necessary for a definition of heat as 'molecular kinetic energy' that is contained within an object. As an exception, Brooker et al., (2010) in their textbook for general biology, state that '[i]n biology, heat is often viewed as energy that can be transferred due to a difference in temperature between two objects or locations' (p. 148).

Mathematical expression/equation: The definition of heat using the First Law of Thermodynamics could be attributed to Born (Canagaratna, 1969). In general, authors of physics textbooks mathematically define the First Law as $\Delta U=Q-W$, but authors of chemistry textbooks define the First Law as $\Delta U=Q+W$ (Ingham 2000). Similarly, Tripp (1976) defines heat in the Journal of Chemistry Education by using the equation $Q=W_{\text {ad }}-W$, whereas in the journal Physics Education, the equation $Q=\Delta U+W$ was cited (Helsdon, 1976). The main differences among these two equations are in the sign and the meaning of $W$ (work done by a system or work done on the system).

Condition/reference frame: The definition of heat in chemistry textbooks may mention the condition of being 'in contact' or 'into contact' (De Berg, 2008). This suggests the process, conduction or convection, in which thermal energy is transferred through objects in physical contact with one another. This condition is not necessary because the transfer of thermal energy is also possible through radiation when objects are not in physical contact.

In general, inconsistency in defining a concept may be understood from the perspective of each of the five common features of definition. For teaching purposes, we should examine whether a definition is internally consistent, and 
seek to reduce variants of definitions of concepts from different scientific disciplines (external consistency), if possible.

The problem of consistency in definition is evidently inextricable from differences in philosophical views. For example, Galili (2001) advocates that weight should be defined as a contact force due to the result of weighing. This is based on the principle of operationalism, which emphasizes the requirement for an operational definition or measurement procedure. In contrast, the definition of weight as a gravitational force may be considered to satisfy the rationalist's canon for a definition.

\subsection{Circularity}

Circular reasoning can be defined as a logical fallacy in which the premise is equivalent to the conclusion (Rips, 2002). An example of a circular definition is: '[e]ntomologist means someone who engages in the science of entomology' (Bassham et al., 2011, p. 101). Such a definiens is not helpful because it consists of a definition that is a slight variation of the word being defined. This kind of circularity can be described as one of self-referencing. To resolve this problem, we may split the scientific term into simpler words such as insects.

However, many problems of circularity have not been satisfactorily resolved. In general, primitive (undefinable) concepts may seem to be a possible solution of the circularity problem. For example, Bunge (2011) proposed that physical concepts such as acceleration, mass and force are undefinable in Newtonian mechanics. We may question the usefulness of undefined concepts when we do not know their precise meaning. It is not trivial whether acceleration should be defined as the rate of change of velocity 'with respect to time' or 'with respect to distance traversed.' Therefore, scientific concepts should be defined in terms of additional scientific terms that are precisely defined, instead of undefined terms. However, if all scientific concepts are defined in terms of each other, it can result in the problem of circularity. The question should be whether the circularity is vicious or non-vicious. We will identify common problems of circularity using the same five categories discussed hitherto. (In the previous definitional problems, we focused on the same concept throughout the section. For circularity, we can only use different scientific concepts to illustrate these five categories more clearly.)

Object/system: Hewitt (2006) defined a neutron as an 'electrically neutral particle that is one of the two kinds of nucleons that compose an atomic nucleus' (p. 771). Within the same textbook, the nucleon is defined as '[a] principal building block of the nucleus. A neutron or a proton' (p. 771). In short, a neutron is defined in terms of a nucleon, and a nucleon is also defined in 
terms of a neutron. Alternatively, the neutron can be defined as a 'nuclear particle of zero charge, spin quantum number $1 / 2$ and a mass of $1.008664904(14) u^{\prime}$ (IUPAC, 2014, p. 989).

Nature/characteristics: Zimmerman and Cuddington (2007) explored students' definitions of the 'balance of nature.' About $40 \%$ of the students provided a circular definition (i.e., 'the way natural systems operate' or similar meanings). Note that the 'balance of nature' is defined with reference to the concept of the 'natural,' and 'natural' is commonly defined in terms of 'nature.' That is, the concept is being defined by means of another term, which is defined in turn by the former concept or its synonyms.

Cause/effect: In biology, a circular notion of the theory of natural selection could be expressed as follows: 'Who survive or who produce more offspring? The fitter! - Who are the fittest? They who survive or leave more offspring!' (Mahner, 1998, p. 412) In this sense, the definition of fitness could be related to the cause of natural selection, and natural selection could result in (or have the effect of) increasing the fitness of a population. In general, a scientific concept can be defined in terms of its effect. However, this 'effect' may be defined by its 'cause,' which is the former concept, resulting in circularity.

Mathematical expression/equation: According to Spurgin (1983), if electric charge is initially defined by the equation, 'electric charge $(q)=$ electric current $(I) \times$ time for which it flows $(t)$,' one should not define electric current as the rate of flow of charge. However, in their textbooks, Wilson, Buffa and Lou (2007) specifically state that 'the electric current is defined as $I=q / t^{\prime}$ (p. 572); this is in contrast to another textbook definition in which '[c] harge is defined as the product of current and time, $q=I \times t^{\prime}$ (Hutchings 200o, p. 258). Textbook writers may determine an acceptable definition by considering either electric charge or electric current to be fundamental, and thus cannot be defined in terms of simpler concepts.

Condition/reference frame: Williams (1999) proposed that the definitions of Newton's laws should include an inertial frame of reference as a condition. However, Brehme (1985) suggested that there is a circularity problem when an inertial frame is defined as any reference frame in which Newton's laws hold. Thus, one does not need to define the inertial frame in terms of Newton's laws. An inertial frame of reference can be defined as a reference frame in which the motion of an observer is not subject to a resultant force and is moving at constant velocity.

The problem of circularity may not be completely eliminable or resolvable. There is always 'inguistic circularity', in that all words in the dictionary are defined by some other words, which will in turn eventually be defined by some of the initially defined words within the same dictionary. Although dictionaries 
have the shortcoming of circularity, this nevertheless does not mean that they are useless (d'Espagnat, 2006).

A more interesting problem is the definitions of mass that have at least three different kinds of circularity related to equations. First, mass $(m)$ is defined in engineering as $W / g$ (Iona, 1975), but weight $(W)$ is defined as $m g$ based on the same equation, $W=m g$. Second, Newton defined mass as the product of the density of the object and its volume $(m=\rho V)$, but density $(\rho)$ is defined by the same equation, $\rho=m / V$. Third, mass is defined in physics textbooks as the ratio of force over acceleration of an object $(m=F / a)$, but force $(F)$ is defined by the same equation, $F=m a$ (French, 1971). In essence, these physical concepts could be defined based on the same equations. However, mass can be mathematically represented as $m=F / a=\rho V=W / g$ showing a circular relationship among these physical concepts. Most importantly, the concept of mass can be verified by multiple operational definitions based on these equations. In a sense, its circularity can become useful because these equations help to link multiple concepts together, and more knowledge can be generated by performing experiments to investigate their relationship.

\section{$4 \quad$ Application of the Framework}

Definitions of scientific concepts may be classified as a theoretical (constitutive) definition or an operational (epistemic) definition. In general, a theoretical definition specifies the meaning by relating it to other scientific concepts and by listing its characteristics, whereas an operational definition is a statement that specifies the concept in terms of the method and conditions of measurement (Galili \& Lehavi, 2006). An important purpose of this framework is to consider how the definitions of a scientific concept can be analyzed and thus be finetuned, instead of producing a superficial definition of the scientific concept. In the following, we will illustrate the use of the framework to provide definitions of heat and weight that are less onerous as compared to complicated concepts such as gene. Next, we will discuss some of the considerations regarding the standards and conventions in defining a scientific concept.

\subsection{Defining Scientific Concepts: Heat and Weight}

To apply this framework, we should determine possible common features of a scientific definition and resolve the related definitional problems. Firstly, to have a comprehensive definition, we may increase the number of common features such that it is adequately comprehensive. Next, for a precise definition, we should ensure that the common features are reasonably precise, and 
all scientific terms used are not ambiguous. Furthermore, to have a consistent definition, we should ensure the common features are internally consistent, and reduce variations among these features in different subjects or disciplines, if possible. Lastly, we should ensure there is no vicious circularity among the five common features; that is, we should avoid self-referencing or circular reasoning in a definition (see Figure 1 for a flow chart of the framework). Alternatively, science educators may recursively review these four definitional problems in different orders until the definition of a scientific concept is considered satisfactory.

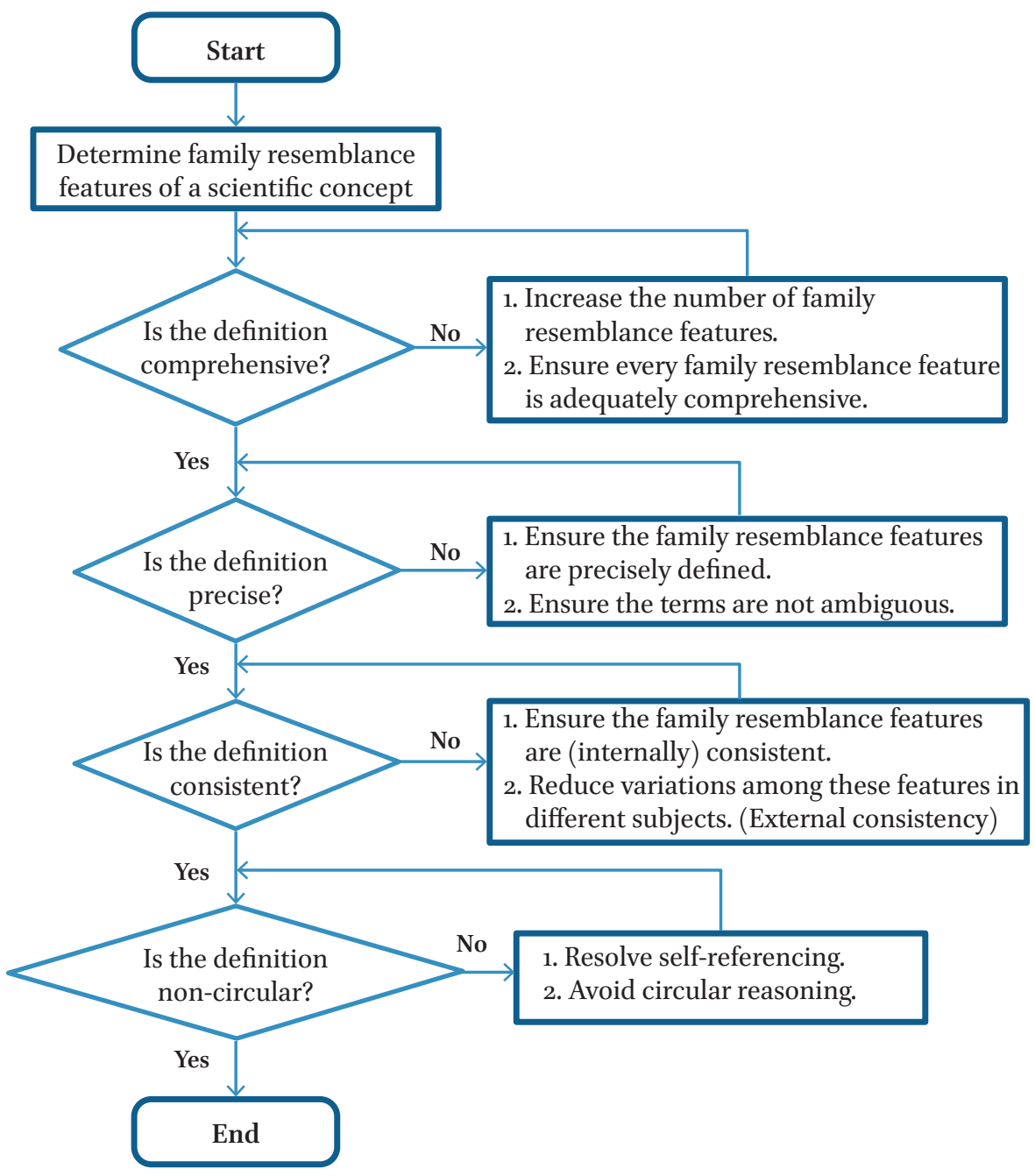

FIGURE 1 A general flow chart of defining scientific concepts 
Based on the discussions in section 3.3, we may determine a preliminary definition of heat by selecting the following features: the system and its surroundings (object/system), transfer of energy (nature/characteristics), temperature difference (cause/effect) and $Q=\Delta U+W$ (mathematical expression/ equation). This definition of heat is more 'comprehensive' than the IUPAC's (2014) definition because it includes an equation. Next, the nature of heat is 'precisely' defined as a transfer of energy; if heat $(Q)$ is also defined as the total kinetic energy of molecules in a body, it can cause confusion because it has a similar meaning as the term 'internal energy' $(U)$ in the equation, $Q=\Delta U+$ $W$. Moreover, there should be no contradiction among the common features of definitions. For example, this definition does not need the condition, in contact, which can result in 'inconsistency'; it is possible to have heat through radiation in a vacuum. Lastly, this definition should not have the problem of 'circularity' in which heat is defined in terms of temperature, and temperature in terms of heat. Thus, by reviewing the four definitional problems, heat can be defined as 'a transfer of energy due to a temperature difference between the system and its surroundings; it is mathematically represented as $Q=\Delta U+W$.' In short, heat occurs at the boundary of a system instead of inside an object. We can represent this theoretical definition of heat in Table 1 below.

We may improve the definition of heat again by using the same framework. For example, we may criticize the definition as being not sufficiently comprehensive because one may include atomic collisions as a microscopic cause of heat. In addition, another condition of heat could be specified as any nonrotating reference frame (DeVoe, 2007).

Similarly, an operational definition of weight can be 'comprehensive' by specifying its object/system as a physical object, nature/characteristics as a contact force, cause/effect as 'not accelerating downward vertically', mathematical expression/ equation as $W=m g$, and condition as a laboratory frame. It should not be 'circularly' defined as 'weight is what bathroom scales read'

TABLE 1 A theoretical definition of heat in tabular form

Concept

Heat

Object/system

Nature / characteristics

Between a system and its surroundings

Cause/effect

Transfer of energy

Mathematical expression/equation

Temperature difference

Theoretically calculated by $Q=\Delta U+W$ 
(Bishop, 1999, p. 239). To be 'precise,' the object/system can also be specified as a calibrated weighing scale. Next, we should check whether the measurement method and condition can be consistently implemented. Thus, we can have an operational definition of weight as follows: 'the weight of a physical object is a contact force (acting downward on the support), and it can be experimentally verified by using the equation, $W=m g$; it is measured by a calibrated weighing scale when it is not accelerating vertically downward in a laboratory frame of reference.' We can represent this operational definition of weight in Table 2 below.

For a theoretical definition of weight, we may initially select the following features: a physical object, gravitational force, gravitational field, $W=m g$, and rotating reference frame. By reviewing the four definitional problems, we can have a theoretical definition of weight as follows: 'the weight of a physical object in a gravitational field is the gravitational force on the object; it can be theoretically calculated using the equation $W=m g$; note that $g$ is the freefall acceleration of the object from the perspective of earth's (rotating) reference frame.'

Essentially, a theoretical definition provides theoretical information of a scientific concept, and an operational definition focuses on the experimental aspects. Although similar features can be found in these two kinds of definition, these features may have different functions. For example, we can theoretically predict the weight of an object using the equation $W=m g$ as specified by a theoretical definition, and we can use the same equation based on an operational definition to calibrate a weighing scale and experimentally verify the concept of weight. However, there should be continuous educational research to investigate the extent to which students can cope with these definitions of scientific concepts.

TABLE 2 An operational definition of weight in tabular form

Concept Weight

Object/system

Nature /characteristics

Cause/effect

Mathematical expression/equation Condition/reference frame
Calibrated weighing scale, physical object Contact force (acting downward on the support)

Not accelerating downward vertically

Experimentally verified by $W=m g$

Laboratory frame of reference 


\subsection{Standards and Conventions in the Light of the Framework}

There should be standards and conventions in defining scientific concepts to improve communication among scientists and to ease learning for students. Based on the framework, the standardization of definitions can be related to object/system, nature/characteristics, cause/effect, mathematical expression/ equation, and condition/ reference frame as suggested below:

Object/system: The IUPAC (2014) defines electric current as the 'base quantity in the system of quantities upon which SI is based' (p. 464). It does not specify the object of electric current. In Garnett and Treagust's (1992) study, students who studied both physics and chemistry tended to have more confusion about the object of electric current, compared with those who only studied chemistry. Some of those dual-discipline students considered electric current as a flow of positive charge (e.g., protons) through metals or a flow of electrons through electrolytic solutions. Garnett and Treagust (1992) propose that the object of electric current be standardized as electrons in a metallic conductor. However, science educators should explain the electron flow and conventional current models as adopted in chemistry and physics respectively.

Nature/characteristics: The IUPAC (2014) defines electron as an '[e]lementary particle not affected by the strong force having a spin quantum number 1/2, a negative elementary charge ...' (p. 477). This definition emphasizes the particle nature of an electron instead of its wave nature. However, physicists may inconsistently use the word 'behave' and 'are' in describing wave-particle duality. Feynman has at least three different descriptions of light or/and electron: 'it really behaves like neither,' 'it is like neither,' and 'they are all "particle waves"' (Feynman et al., 1963, p. 37-1). Science educators should stress the difference between the words 'behave' and 'are.' When the word 'behave' or 'are' is used, there should be a clarification of whether the concept is a mathematical construct or an instrumental construct.

Cause/effect: The IUPAC (2014) defines heat as 'energy transferred from a hotter to a cooler body due to a temperature gradient.' The cause of heat being a temperature gradient can seem abstract to students. However, the cause of heat could be classified as microscopic and macroscopic. For example, Hecht (2003) defines heat as 'the thermal energy transferred, via atomic collisions' (p. 459). That is, the microscopic cause of heat can be specified as atomic collisions, and the macroscopic cause can be a temperature difference. On the other hand, the effect of heat can be a temperature difference and phase change. Therefore, it is possible to have operational (calorimetric) definitions of heat based on this measurable effect. In general, the theoretical definition 
of a scientific concept may be standardized by specifying its cause, and the operational definition should include the observable effect.

Mathematical expression/equation: The IUPAC (2014) defines magnetic field strength $(H)$ as 'magnetic flux density divided by the permeability' (p. 875). Moreover, the IUPAC (2014) defines magnetic flux density (or magnetic induction, $B$ ) as 'a vector characterizing a magnetic field' (p. 875). Mathematically, magnetic flux density can be represented as $B=\mu \mathrm{H}$. In physics, there are multiple definitions of magnetic field. Jackson (1999) defines magnetic field as $H$-field and magnetic induction as $B$-field, but other textbook authors may define magnetizing field as $H$-field and magnetic field as $B$-field. In short, magnetic field is either defined as $H$-field or $B$-field. Interestingly, Feynman et al. (1964) described the mathematical definitions of a magnetic field as 'horrible' because there were many different representations due to different names, notations, and systems of units. Thankfully, more equations are now defined in terms of the International System of Units (SI).

Condition/reference frame: The IUPAC (2014) includes the following conditions in the definitions of various scientific concepts: well-defined conditions, specific local environmental conditions, experimental condition, analytical condition, specified conditions, controlled conditions, measurement conditions, pathological conditions, physiological conditions, equilibrium conditions, electronic condition, and extreme physico-chemical conditions. Note that these conditions are not always exactly specified and can be either singular or plural. Some of the conditions were arbitrarily defined and could be redefined in the future. As an example, the IUPAC (2014) defines standard conditions for gases as ' $\left[\mathrm{t}\right.$ ] emperature, $273.15 \mathrm{~K}\left(\mathrm{o}^{\circ} \mathrm{C}\right)$ and pressure of $10^{5}$ pascals. IUPAC recommends that the former use of the pressure of $1 \mathrm{~atm}$ as standard pressure (equivalent to $1.01325 \times 10^{5} \mathrm{~Pa}$ ) should be discontinued' (p. 1435). It is important to standardize these conditions such that scientific experiments can be reproducible under the same test conditions.

It would be a challenge to maintain standards and conventions in the definitions of scientific concepts. For example, the IUPAC does not specify any condition in the definition of heat. On the contrary, some chemistry textbooks define heat in terms of conditions such as 'in contact' or 'into contact.' However, scientists may prefer some flexibility in having alternative definitions for the purposes of research or critique in different subject disciplines or research communities. More importantly, there should be continued communication among scientists and science educators to improve definitions of scientific concepts for learning. 


\section{Limitations and Implications}

\subsection{Limitations of the Framework}

One limitation of this framework is that it includes only five common features of definitions. Note that a definition is affiliated with a scientific law, theory, or model. For example, the concept of an electron can be related to the classical electron theory, Dirac electron theory, or quantum field theory. Essentially, this feature could be specified as law/theory/model. However, the concept of mass in the special theory of relativity could be mathematically defined as being velocity dependent or Lorentz invariant. Thus, there can be at least two different systems of mathematical definitions or equations even within a scientific theory.

On the other hand, another possible definitional problem for defining scientific concepts is comprehension. A definition of mouse as 'a quadrupedal mammalian of any of the more diminutive species of the genus Mus of the order Rodentia' (Bassham et al., 2011, p. 101) is likely to be confusing to elementary students. There should be empirical evidence to support appropriate definitions that are progressively determined for students from different age groups and backgrounds. However, students' abilities to cope with these definitions are also dependent on science educators' pedagogical approaches.

Another limitation of this framework is that there may be disagreement on the definition of definition. We can define a definition as a determination of a concept (Rickert, 1888); others define it as a sign-sign correspondence (Bunge, 1998), an explanation of the meaning of a word (Belnap, 1993), or 'a convention, hence neither true nor false but at most practical or impractical, convenient or inconvenient' (Mahner, 1998, p. 415). It is also possible to have disagreements about whether a definition is sufficiently comprehensive, precise, and consistent. Furthermore, there may not be consensual definitions of theoretical definition and operational definition. Hence, there should be further research with regard to the nature of definition and its implications to learning.

\subsection{Implications of the Framework}

It is important to point out that students and teachers struggle in understanding some scientific concepts because of difficulties in defining the terms. For example, it is difficult to define the concept of gene because of new discoveries (Portin, 2009). There are also studies that found students and teachers had difficulties understanding the concept of gene, and distinguishing genes and alleles (e.g., Dikmenli et al., 2011; Pashley, 1994). Students' understanding is also not enhanced by a simple definition of an allele as 'a variant form of a gene.' 
Thus, the framework of defining scientific concepts has implications for teachers in universities, high schools, and elementary schools.

For university lecturers, it should not be difficult to apply the two-level framework (five common features and four definitional problems) presented here. However, it is impractical for university lecturers to continuously discuss the definitions of many scientific concepts and analyze definitional problems in every lesson. As misunderstandings of definitions can have a significant impact on learning, university lecturers should minimally lead students to discuss definitional problems of key scientific concepts found in a given module at the beginning of the semester. Better still, they should develop multiple representations of the five common features, such as verbal, diagrammatic, and symbolic representations

High school teachers could be trained to apply a simplified framework that focuses on three common features and three definitional problems. In this framework, the three main common features are object/system, nature/characteristics, and cause/effect; high school teachers may include mathematical expression/equation or/and condition/reference frame when they are the essential features for certain scientific concepts. The three main definitional problems are comprehensiveness, precision, and consistency; we can exclude circularity as it may be too abstract for students. Essentially, the learning of definitions at the high school level should emphasize the recognition of the common features (or patterns of definitions) instead of rote memorization.

Elementary school teachers could be trained to apply a further simplified framework in which they need not learn about definitional problems. However, there will be four common features: object/system, nature/characteristics, cause/effect, and classification/category. The feature classification is useful because it is relatively more common in biology and elementary science topics. For instance, a whale is a mammal instead of a fish; in general, a concept may be classified according to a certain category or sub-category. However, elementary definitions should have only one or two common features to reduce memory loads of students. There should also be educational research to determine the extent to which elementary school teachers can cope with existing definitions.

In the future, the common features of definitions can vary systematically for students at different educational levels. In elementary schools, students can be provided with an elementary definition of weight as a force of gravity. Elementary teachers may focus on only two features of weight: force (nature/ 
characteristics) and gravity (cause/effect). Note that weight is defined using simple and short words: force and gravity. In high schools, science teachers can replace the term force of gravity with gravitational force. In addition, the definition of weight can be explained in more details such as a gravitational field of the earth (cause/effect) and $W=m g$ (equation). Thus, high school students can use the equation to calculate the change in weight due to the change in the gravitational field. In colleges or universities, lecturers can guide students to discuss or debate different definitions of weight: gravitational force and contact force. Furthermore, the concept of weight can be introduced with more details from the perspective of object/system, cause/effect, mathematical expression/ equation, and condition/reference frame as compared to definitions proposed for elementary and high schools (see Table 3). More importantly, the learning of definitions can be more effective if students are guided to reconstruct scientific concepts or analyze their definitions (Wong, Chu, \& Yap, 2014).

TABLE 3 Possible features of definition (weight) for students from different educational levels

\begin{tabular}{|c|c|c|c|}
\hline Features & $\begin{array}{l}\text { Elementary } \\
\text { School }\end{array}$ & $\begin{array}{l}\text { High } \\
\text { School }\end{array}$ & University \\
\hline Object/system & & & $\begin{array}{l}\text { System (an object and } \\
\text { earth) Celestial body }\end{array}$ \\
\hline $\begin{array}{l}\text { Nature/ } \\
\text { characteristics }\end{array}$ & $\begin{array}{l}\text { Force } \\
\text { (of gravity) }\end{array}$ & $\begin{array}{l}\text { Gravitational } \\
\text { force }\end{array}$ & $\begin{array}{l}\text { Resultant gravitational } \\
\text { force } \\
\text { Contact force }\end{array}$ \\
\hline Cause/effect & Gravity & $\begin{array}{l}\text { Gravitational } \\
\text { field }\end{array}$ & $\begin{array}{l}\text { Gravitational field } \\
\text { Acceleration of an } \\
\text { elevator }\end{array}$ \\
\hline $\begin{array}{l}\text { Mathematical } \\
\text { expression/equation }\end{array}$ & & $W=m g$ & $\begin{array}{l}W=G M m / r^{2} \\
W=m g-m \omega^{2} R\end{array}$ \\
\hline $\begin{array}{l}\text { Condition/reference } \\
\text { frame }\end{array}$ & & & $\begin{array}{l}\text { Static equilibrium } \\
\text { Rotating frame of } \\
\text { reference }\end{array}$ \\
\hline
\end{tabular}


Scientists and science educators may have individual preferences regarding how they define scientific concepts, with recourse to common features such as object/system, nature/characteristics, cause/effect, mathematical expression/ equation, and condition/reference frame. Based on the framework, scientific concepts can first be related to an object/system, and its nature/characteristics or properties should be specified. In addition, the feature cause/effect could be included with at least a condition or reference frame. If possible, we should specify a mathematical expression or equation in which the concept can be verified precisely.

Scientific concepts can be analyzed and defined by means of at least the following considerations: comprehensiveness, precision, consistency, and circularity. First, a definition should be made comprehensive by including more features of definitions, but it can be overwhelming to have too many details in every feature. Next, the common features of definition should be reasonably precise; but nevertheless, the inclusion of a mathematical equation does not mean the definition to be completely precise. Third, while a definition may not be externally consistent when it is compared to another definition from another field, it should be internally consistent with respect to its common features. Lastly, we should avoid self-referencing (vicious circularity) in defining scientific concepts; but Bunge's (1998) vision of primitive concepts has not resolved the problem of circularity.

It should be possible to develop a more sophisticated three-level framework by incorporating additional considerations. Currently, science educators can first apply this two-level framework to the definition of scientific concepts, and we may consider a third level which specifies a philosophical position. It may help us to redefine a scientific concept, whether it is a mathematical construct, an instrumental construct, or a real entity.

In summary, we hope that science educators would find the framework useful as a guide to remind them of the possible features of definitions instead of working from scratch, and to reduce issues of comprehensiveness, precision, consistency, and circularity. On the other hand, scientists need to continuously redefine science concepts for research purposes based on new discoveries, but they may use the framework to provide definitions for the public. More important, students (especially students in Asian countries where education systems have long promoted rote memorization) can apply the framework to construct and make sense of science definitions from the perspectives of an object, characteristics, cause/effect, equation, or condition in the definitions instead of memorizing the definitions. In the future, we hope to see more 
studies exploring the potential for this framework to be employed in place of pedagogical strategies that emphasize memorization only. Lastly, there should be more communications among educational researchers and science educators from different disciplines on common definitions for students and using this framework could be a good start.

\section{Abbreviation}

IUPAC International Union of Pure and Applied Chemistry

\section{Ethical Considerations}

The data collected from this article did not involve human subjects, so no human subjects approval was necessary.

\section{About the Authors}

Chee Leong Wong is an educational consultant in Singapore. He taught physics for more than 10 years in Catholic Junior College and National University of Singapore High School of Mathematics and Science. He completed his $\mathrm{PhD}$ thesis in Nanyang Technological University, Singapore, titled "A framework for defining physical concepts". His research interests include multiple representations in science education, inquiry approaches in learning science, definitions of scientific concepts, history and philosophy of science, and analyzing The Feynman Lectures on Physics.

Hye-Eun Chu is a senior lecturer in science education at Macquarie University in Sydney, Australia. Before joining Macquarie University, she was an assistant professor in science education for 6 years at Nanyang Technological University, Singapore. Her research interests include the investigation of students' conceptual development in science learning with a current focus on students' explanatory models, the influence of learner belief on science learning, and on-going assessment in the context of inquiry-based teaching.

Kueh Chin Yap is a retired Science and Physics Education Associate Professor at the National Institute of Education, Nanyang Technology University Singapore. His research interests include alternative conceptions in science/ 
physics, multiple representations in science/physics teaching and learning, visual thinking in science/physics teaching and learning, integration of ICT in science/physics teaching and learning, scientific inquiry and processes in investigative problem solving, and assessment in science/physics for learning.

\section{References}

Alonso, M. (1990). Comment I by Alonso. The Physics Teacher, 28(7), 441.

Arunan, E., Desiraju, G. R., Klein, R. A., Sadlej, J., Scheiner, S., Alkorta, I., Clary, D. C., Crabtree, R. H., Dannenberg, J.J., Hobza, P., Kjaergaard, H. G., Legon, A. C., Mennucci, B., \& Nesbitt, D. J. (2011). Defining the hydrogen bond: An account. Pure and Applied Chemistry, 83(8), 1619-1636.

Baierlein, R. (1990). The meaning of temperature. The Physics Teacher, 28(2), 94-96.

Bassham, G., Irwin, W., Nardone, H., \& Wallace, J. M. (2011). Critical Thinking: a student's introduction (4th ed.). Boston: McGraw-Hill.

Belnap, N. (1993). On rigorous definitions. Philosophical Studies, 72(2/3), 115-146.

Benfey, O. T. (2006). The conceptual structure of the sciences: Reemergence of the human dimension. In Baird, D., Scerri, E., \& McIntyre, L. (2006). Philosophy of chemistry: Synthesis of a new discipline. Dordrecht: Springer.

Bishop, R. (1999). Weight - An accurate, up-to-date, Layman's Definition. The Physics Teacher, 37(4), 238-239.

Brehme, R. W. (1985). On force and the inertial frame. American Journal of Physics, 53(10), 952-955.

Brooker, R. J., Widmaier, E. P., Graham, L. E., Stiling, P. D., Hasenkampf, C., Hunter, F. F., Bidochka, M., \& Riggs, C. D. (2010). Biology (Canadian ed.). New York, NY: McGraw-Hill Ryerson.

Bunge, M. (1998). Philosophy of science: From problem to theory, Vol. 1. New Jersey: Transaction Publishers.

Bunge, M. (2011). Knowledge: Genuine and bogus. Science \& Education, 20(5-6), $411-438$.

Campbell, N. A., \& Reece, J. B. (2008). Biology (8th ed.). San Francisco, CA: Pearson Benjamin Cummings.

Canagaratna, S. G. (1969). Critique of the definitions of heat. American Journal of Physics, 37(7), 679-683.

Cassels, J. R. T., \& Johnstone, A. H. (1985). Words that matter in science. London: Royal Society of Chemistry.

Coelho, R. L. (2010). On the concept of force: How understanding its history can improve physics teaching. Science \& Education, 19(1), 91-113. 
De Berg, K. C. (2008). The concepts of heat and temperature: The problem of determining the content for the construction of an historical case study which is sensitive to nature of science issues and teaching-learning issues. Science \& Education, $17(1), 75^{-114}$.

d'Espagnat, B. (2006). On physics and philosophy. Princeton: Princeton University Press.

DeVoe, H. (2007). Particle model for work, heat, and the energy of a thermodynamic system. Journal of Chemical Education, 84(3), 504-512.

Dikmenli, M., Cardak, O., \& Kiray, S. A. (2011). Science student teachers' ideas about the 'gene' concept. Procedia-Social and Behavioral Sciences, 15, 26o9-2613.

Doige, C. A., \& Day, T. (2012). A typology of undergraduate textbook definitions of 'heat' across science disciplines. International Journal of Science Education, 34(5), $677-700$.

Duit, R. (2014). Teaching and learning the physics energy concept. In J. Krajcik, R. F. Chen, A. Eisenkraft, D. Fortus, K. Neumann, J. C. Nordine, \& A. Scheff, Eds. Teaching and learning of energy in K-12 education. Dordrecht: Springer.

Einstein, A. (1921). Geometry and experience. In Ideas and opinions (pp. 232-246). New York: Bonanza.

Feynman, R. P., Leighton, R. B., \& Sands, M. (1963). The Feynman lectures on physics vol I. Reading, MA: Addison-Wesley.

Feynman, R. P., Leighton, R. B., \& Sands, M. (1964). The Feynman lectures on physics vol II. Reading, MA: Addison-Wesley.

French, A. P. (1971). Newtonian mechanics. New York: W. W. Norton.

Galili, I. (2001). Weight versus gravitational force: historical and educational perspectives. International Journal of Science Education, 23(10), 1073-1093.

Galili, I., \& Lehavi, Y. (2006). Definitions of physical concepts: A study of physics teachers' knowledge and views. International Journal of Science Education, 28(5), 521-541.

Garnett, P. J., \& Treagust, D. F. (1992). Conceptual difficulties experienced by senior high school students of electrochemistry: Electric circuits and oxidation-reduction equations. Journal of Research in Science Teaching, 29(2), 121-142.

Gayford, C. G. (1986). Some aspects of the problems of teaching about energy in school biology. European Journal of Science Education, 8(4), 443-450.

Giancoli, D. C. (2005). Physics: Principles with applications (6th ed.). Upper Saddle River, NJ: Prentice Hall.

Giordano, N. J. (2010). College physics. Reasoning and relationship. Belmont, CA: Cengage Brooks-Cole.

Groves, F. H. (1995). Science vocabulary load of selected secondary science textbooks. School Science and Mathematics, 95(5), 231-235.

Halliday, D., Resnick, A., \& Walker, J. (2001). Fundamentals of physics (6th ed.). New York: Wiley. 
Harwood, T. D. (2009). The circular definition of populations and its implications for biological sampling. Molecular Ecology, 18(5), 765-768.

Hecht, E. (2003). Physics: Algebra/trigonometry (3rd ed.). Pacific Grove, California: Brooks/Cole Publishing.

Helsdon, R. M. (1976). Teaching thermodynamics. Physics Education, 11(4), 261-262.

Hewitt, P. (2006). Conceptual physics (1oth ed.). San Francisco: Addison-Wesley.

Huang, P. L. (2009). A comprehensive definition for metabolic syndrome. Disease Models \& Mechanisms, 2(5-6), 231-237.

Humphreys, P. W. (1999). Causation. In W. H. Newton-Smith (ed.), A Companion to the philosophy of science (pp. 31-40). Blackwell: Oxford.

Hutchings, R. (2000). Physics (2nd ed.). Cheltenham: Nelson.

Ingham, W. H. (2000). A consistent sign convention for work. The Physics Teacher, $38(3), 160-161$.

Iona, M. (1975). The meaning of weight. The Physics Teacher, 13(5), 263-274.

Iona, M. (1999). Weight - An official definition. The Physics Teacher, 37(4), 238.

IUPAC (2014). Compendium of chemical terminology (Version 2.3.3). Compiled by A. D. McNaught \& A. Wilkinson. Oxford: Blackwell Scientific Publications. Retrieved October 8, 2014 from http://goldbook.iupac.org/.

Jackson, J. D. (1999). Classical electrodynamics (3rd ed.). New York: Wiley.

Kaya, E., \& Erduran, S. (2013). Integrating epistemological perspectives on chemistry in chemical education: The cases of concept duality, chemical language, and structural explanations. Science \& Education, 22(7), 1741-1755.

Keller, E. F. (2005). The century beyond the gene. Journal of Biosciences, 30(1), 3-10.

Khine, M. S. (Ed.). (2015). Science education in East Asia: Pedagogical innovations and research-informed practices. Springer.

Lancor, R. (2014). Using metaphor theory to examine conceptions of energy in biology, chemistry, and physics. Science \& Education, 23(6), 1245-1267.

Laszlo, P. (1999). Circulation of concepts. Foundations of Chemistry, 1(3), 225-238.

Laszlo, P. (2013). Towards teaching chemistry as a language. Science \& Education, 22(7), 1669-1706.

Mahner, M. (1998). Operationalist fallacies in biology. Science \& Education, 7(4), 403-421.

Margenau, H. (1942). The role of definitions in physical science, with remarks on the frequency definition of probability. American Journal of Physics, 10(5), 224-232.

Morrison, R. C. (1999). Weight and gravity - The need for consistent definitions. The Physics Teacher, $37(1), 5^{1-52}$.

Pascal, B. (1657). The spirit of geometry. In J. C. Sager (ed.) (200o). Essays on definition (pp. 95-107). Amsterdam: John Benjamins B.V. 
Pashley, M. (1994). A-level students: their problems with gene and allele. Journal of Biological Education, 28(2), 120-126.

Portin, P. (2009). The elusive concept of the gene. Hereditas, 146(3), 112-117.

Pushkin, B. D. (1997). Scientific terminology and context: How broad or narrow are our meanings? Journal of Research in Science Teaching, 34(6), 661-668.

Quílez, J. (2009). From chemical forces to chemical rates: A historical/philosophical foundation for the teaching of chemical equilibrium. Science \& Education, 18(9), 1203-1251.

Rickert, H. (1888/200o). The theory of definitions. In J. C. Sager (ed.) Essays on definition. (pp. 191-249.) Amsterdam: John Benjamins B.V.

Rips, L. J. (2002). Circular reasoning. Cognitive Science, 26(6), 767-795.

Romer, R. H. (2001). Heat is not a noun. American Journal of Physics, 69(2), 107-109.

Ruchlis, H. (1964). How precise shall we be? The Physics Teacher, 2(4), 175.

Serway, R. (2003). College physics (6th ed.). California: Thomson Learning.

Spurgin, C. B. (1983). Definitions of quantities and units. Physics Education, 18(6), 259-262.

Strömdahl, H. R. (2012). On discerning critical elements, relationships and shifts in attaining scientific terms: The challenge of polysemy/homonymy and reference. Science \& Education, 21(1), 55-85.

Suppe, F. (2000). Definitions. In W. H. Newton-Smith (ed.), A companion to the philosophy of science (pp. 76-78). Blackwell: Oxford.

Suppes, P. (1957). Introduction to logic. Princeton, NJ: D. Van Nostrand.

Swendsen, R. H. (2011). How physicists disagree on the meaning of entropy. American Journal of Physics, 79(4), 342-348.

Trifonov, E. N. (2012). Definition of life: Navigation through uncertainties. Journal of Biomolecular Structure \& Dynamics, 29(4), 647-65o.

Tripp, T. B. (1976). The definition of heat. Journal of Chemistry Education, 53(12), 782-784.

Williams, H. T. (1999). Semantics in teaching introductory physics. American Journal of Physics, 67(8), 670-680.

Wilson, J. D., Buffa, A. J., \& Lou, B. (2007). College physics (6th ed.). New Jersey: Pearson. Wittgenstein, L. (1953). Philosophical investigations (G. E. M. Anscombe, Trans.). Oxford: Blackwell.

Wong, C. L. (2014). A framework for defining physical concepts. Ph.D. Thesis. Nanyang Technological University.

Wong, C. L., Chu, H. E., \& Yap, K. C. (2014). Developing a framework for analyzing definitions: A study of the Feynman Lectures. International Journal of Science Education, $36(15), 2481-2513$. 
Zemansky, M. W., \& Dittman, R. H. (1981). Heat and thermodynamics: An intermediate textbook, 6th ed. Singapore: McGraw-Hill.

Zimmerman, C., \& Cuddington, K. (2007). Ambiguous, circular and polysemous: students' definitions of the "balance of nature" metaphor. Public Understanding of Science, 16(4), 393-406. 